\title{
System of trustworthy deformational precursors of highly stressed rock samples failure
}

\author{
V.V. Makarov The Far Eastern Federal University, Russia
}

L.S. Ksendzenko The Far Eastern Federal University, Russia

V.v. Gnitienko The Far Eastern Federal University, Russia

A.M. Golosov The Far Eastern Federal University, Russia

N.A. Opanasiuk The Far Eastern Federal University, Russia

A.A. Opanasiuk The Far Eastern Federal University, Russia

\section{Abstract}

The phenomena of anomalous deformation and failure of rock specimens under uniaxial compression have been studied in a laboratory.

A system of trustworthy deformational precursors of the failure stage was developed. The system includes long-term, middle-term and short-term precursors. The threshold of dilatancy and the turning point of the deformational curve are recognised as long-term precursors. The middle-term precursor is determined as a point of the increment sign change of the specific volume deformation. The short-term precursor is characterised by the specific volume deformation increments jump.

The acoustic emission research method had been used to control the deformational and failure process. There was a tight correlation between the deformational precursors system of failure and the mesocracking process under the loading.

The mathematical model of self equilibrium stresses had been successfully used to describe the anomalous deformations distribution.

\section{Introduction}

The phenomena of massif rock failure has become a global ecological threat in the recent years. The 2010 earthquakes in Haiti reached a magnitude of 7, and in Japan in 2011 a magnitude of 9). These earthquakes have resulted not only in significant human losses and unprecedented human tragedy, but also threats of epidemics and radioactive pollution across the globe. Despite the existence of extensive networks of seismoacoustic stations and a huge number of scientific works, prediction of such events remains impossible (Sagiya et al., 2011). This stems from an insufficient understanding of the destructive mechanisms in geomaterials at various structural levels. There is a need for an urgent departure from traditional conservative schemes of the prediction of such catastrophic events. Insubstantial scientific effort has been invested to develop new conceptual approaches towards the definition of critical conditions of geodynamic systems.

According to new theoretical concepts, the rock mass can be analysed as a block hierarchical medium, in which the elements demonstrate self similar fractal characteristics on different hierarchical levels (Makarov, 2004). Laboratory methods on rock samples may be successfully used for forecasting failure in rock mass. Development of a trustworthy system of precursors, including the long-term, middle-term and short-term, is an extremely important geodynamical task.

Deformational precursors occupy a prominent position over all another methods because their specific properties are methods of direct control. At the same time, indirect methods such as acoustic emission, 
have been widely used in laboratory research and can characterise the cracking process in rocks (Lockner et al., 1991). The complex method and system of criterions are more successful for use in geodynamical forecasting.

Laboratory research of rock samples destruction has the advantage that detailed measurements can be made by eliminating the factors of heterogeneity which exist in a rock mass. Thus those criteria which have unequivocal interpretation will be reliable. In the presented work, the system of reliable deformation precursors of the rock samples destruction, corresponding to formation stages of the dissipative mesocracking structures, is confirmed by a fixed acoustic method.

The mathematical model of the defective medium applied to the description of deformation anomalies determined in the experiments, considers the occurrence in the sample of rock a field of self-counter balanced pressure caused by the formation and development of the dissipative mesocracking structure before destruction.

\section{Experimental research of the deformational relationships of rock samples in the prefailure area of loading}

The process of rock samples macrodestruction proceeds in stages, which are initially characterised, by a preparatory one of uniform material damage under the influence of the compressive stress. This is followed by localisation of mesocracks as the focus of the macrorupture formation. At the last stage, there is a macrodefect development (Lockner et al., 1991).

On the basis of general laws, deformation research should consider stages of the macrofailure development process and not be limited to control in the central part of the sample, and local (in one to two points) arrangement of the gauges. Therefore, experiments by definition of the rock sample deformation laws at compression, in loading in the prefailure area have been done under multidot schemes, both horizontal and vertical directions. To determine the character of rock sample deformation research was undertaken using cylindrical samples. The relation of height to diameter is accepted as $\mathrm{h} / \mathrm{d}=2$ according to recommendations of Guzev and Makarov (2007).

The compression force was applied by a servocontrole loading device MTS-816 with a constant speed of deformation before strength achievement (Mechanics Test Systems, 2009). Tensoresistors were used for deformation measurement and these allowed definition of local deformations, both in the central part of the sample and over its height. Research was undertaken on various rock types, including dacites, granodiorite, granity-porphyry and others. Measurement of deformations was undertaken not only in a separate part of the sample, but also around its entire perimeter. Tensoresisters were pasted in various schemes. Label of gauges schemes and the samples prepared for experiment are presented in Figure 1. Indications of the tensoresisters were fixed by means of device UIU-2002 software in an automatic mode.
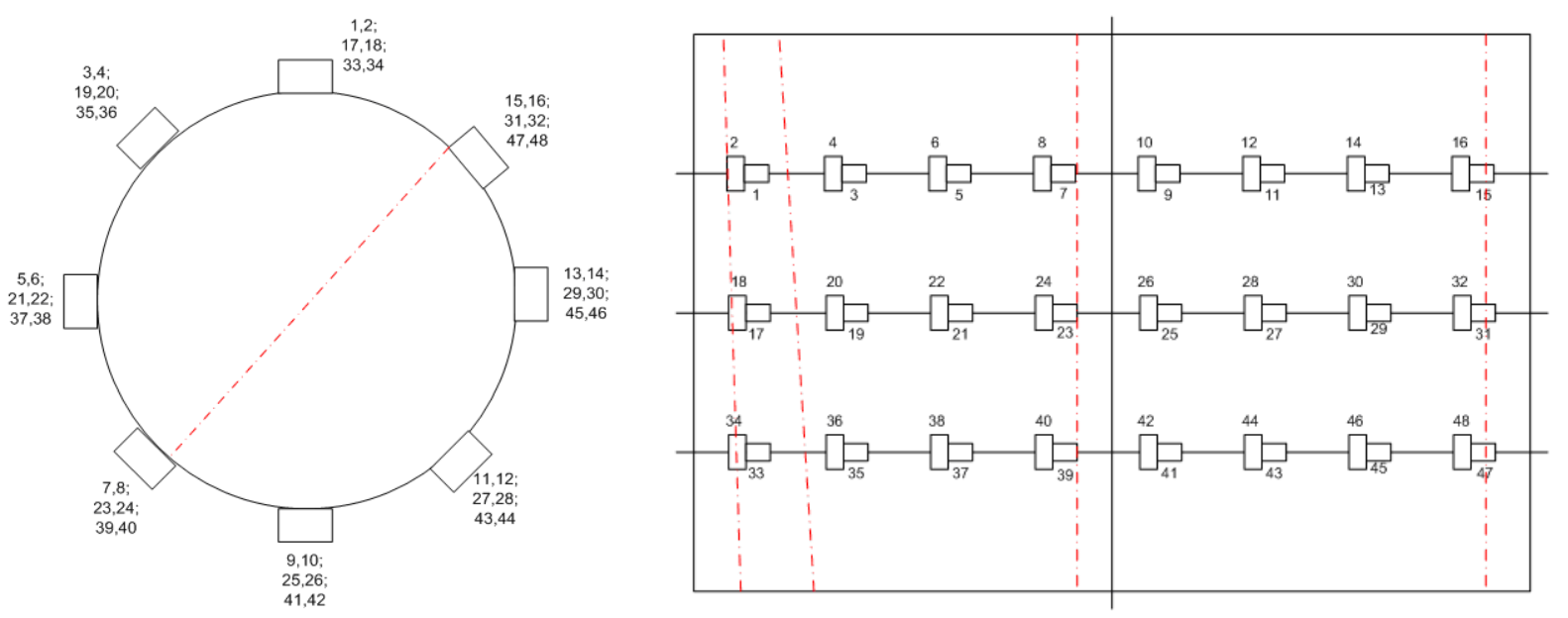

Figure 1 Measurement scheme and macrocracks directions 
Research has been carried out in the Laboratory of Geodynamics, FEFU. In Figure 2 the laboratory used for the testing is shown. In Figure 3 samples prepared for testing are also shown. In total 4 series of 10 samples in each of the rock types have been tested. Results were provided for uniaxial compression of rocks samples conditions under the multidot scheme of measurements, with the quantity of measuring points varying from 4 to 24 (Figure 3). Figure 4 shows a failed sample of dacite.

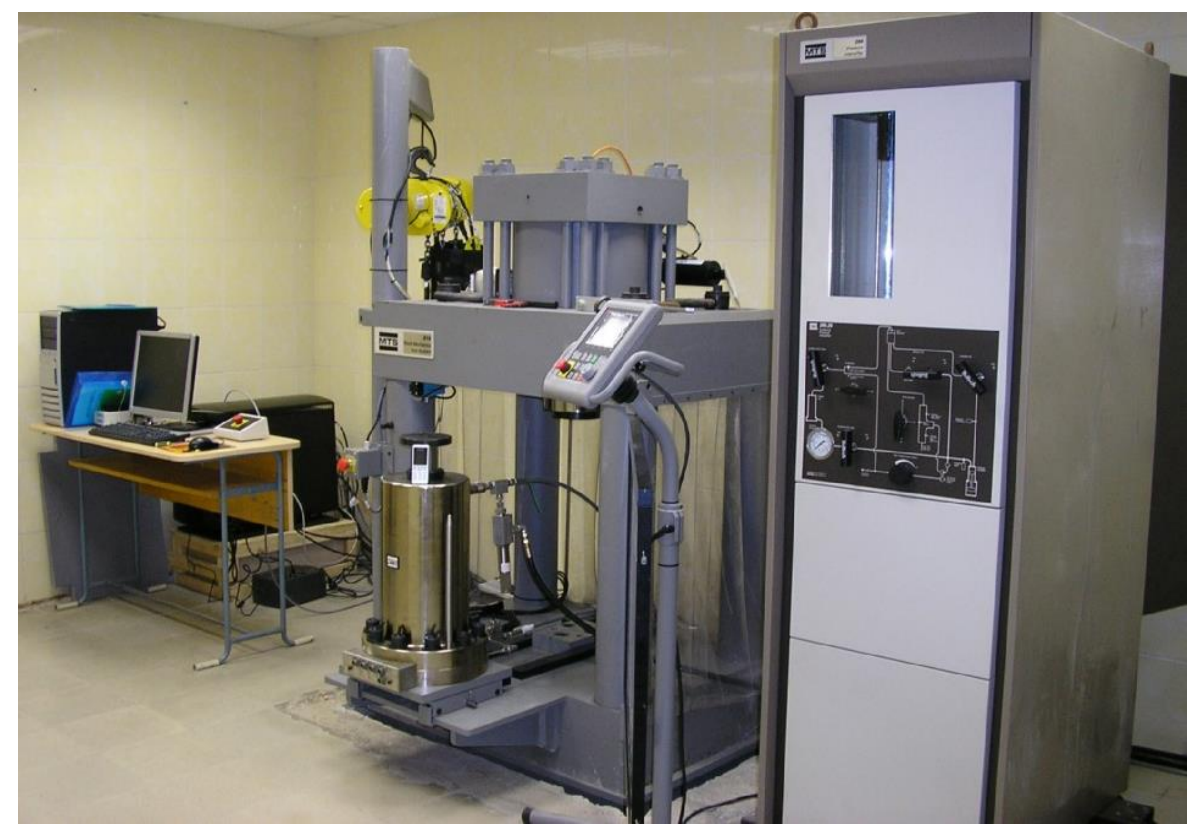

Figure 2 MTS-816 load system in Laboratory on Geodynamics, FEFU

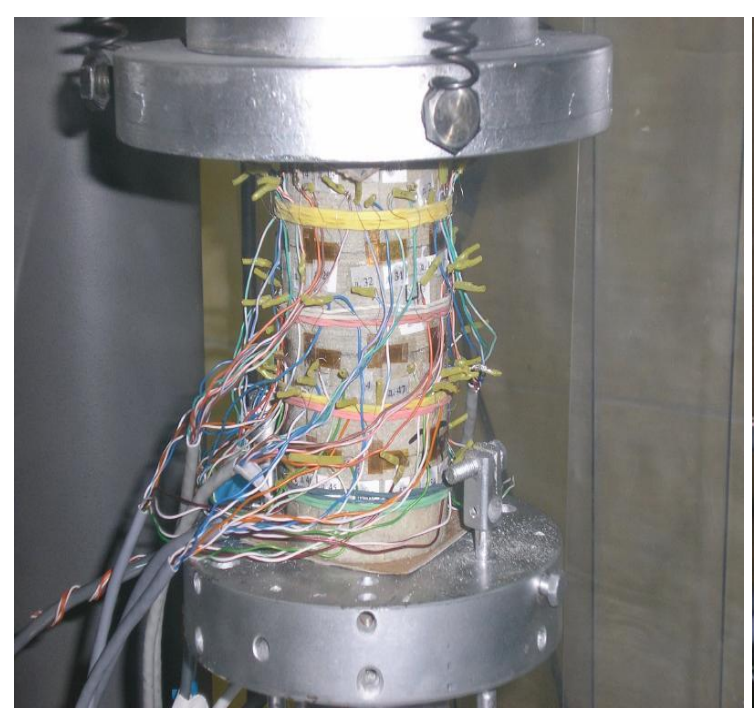

a)

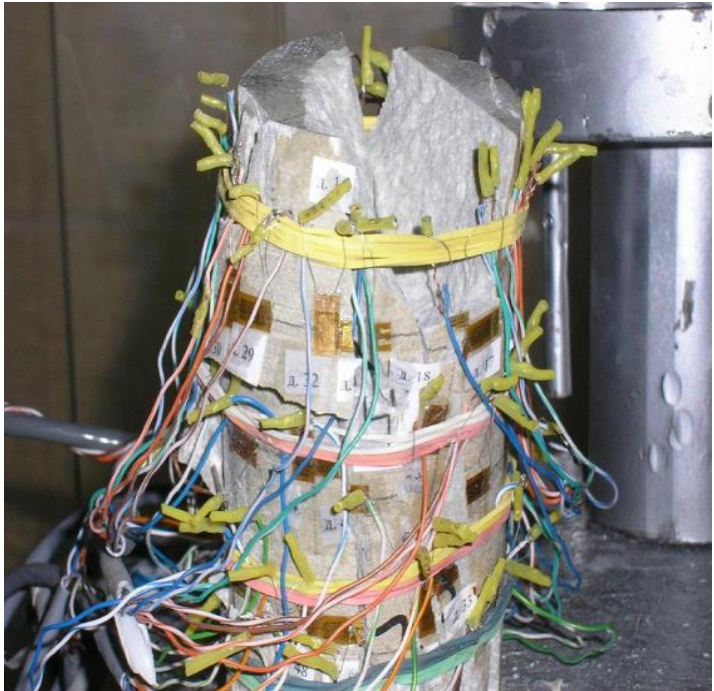

b)

Figure 3 Rock sample before (a) and after (b) failure

Let us consider some results of rocks samples deformation research in a prefailure condition with use of the high stiffness and servo-controlled loading system. 


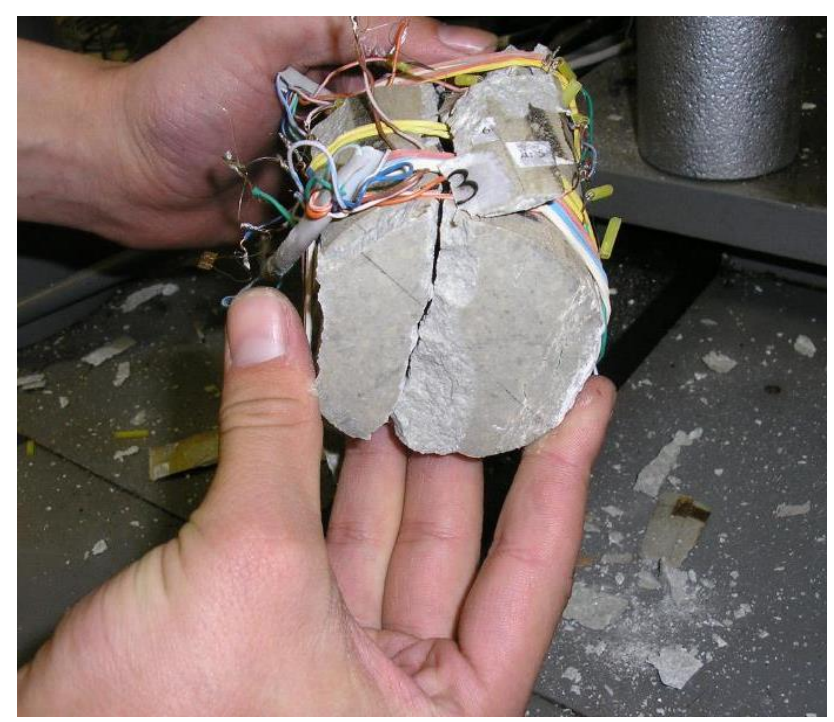

Figure 4 Macrofailure character of dacite sample (No. 3)

Deformation curves of linear deformations for the central part of the sample are shown in Figure 5. It is seen that in the prefailure stage of loading flattening of the curves can be observed with a corresponding reduction in the modulus of deformations from 1.5 to 3 times. The cases of a reversal of the linear deformations are also marked.

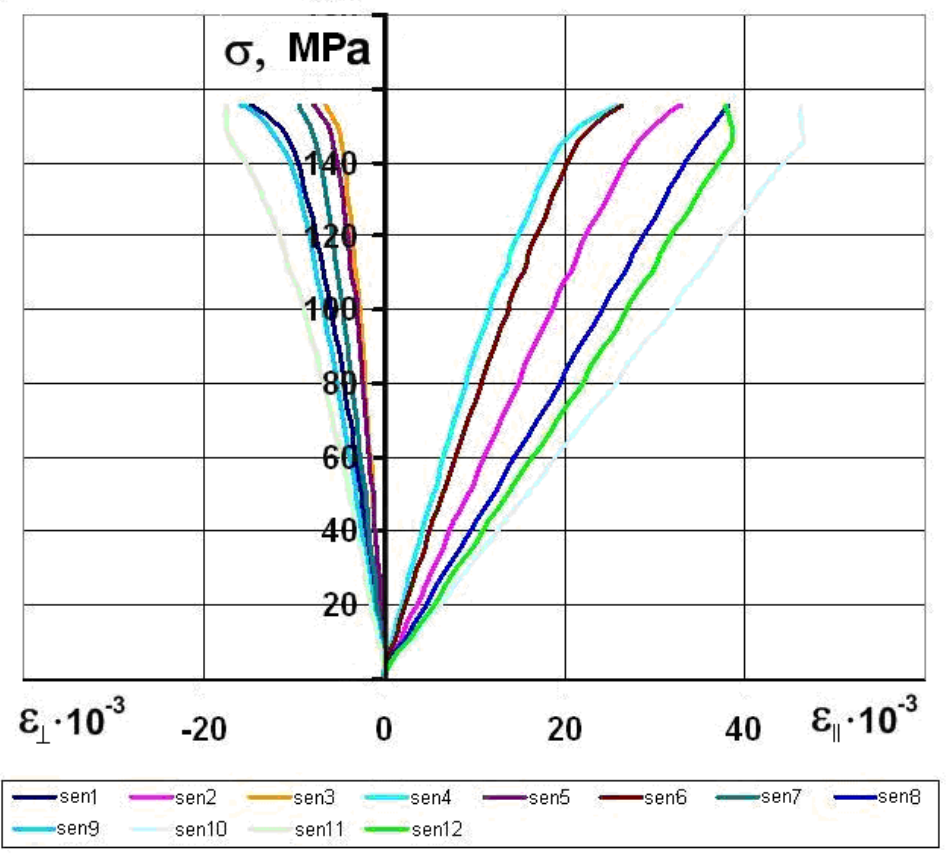

Figure 5 Relationships of rock samples deformation in prefailure stage of loading: I inear deformations in central part

In Figures 6 and 7 the typical picture of distribution of the specific volume deformations on the perimeter of the sample in its central part in the prefailure area of loading are shown. Specific volume deformations are considered in connection with the necessity for the reduction of the volume deformation increment values to unit pressure, and corresponding to a comparable loading curve at all stages of deformation:

$$
\varepsilon_{v}^{s p}=\Delta \varepsilon_{v} / \Delta \sigma, \mathrm{MPa}
$$


It can be seen that the process of decompaction is altered by the sudden change in the increments of deformation of a different sign, followed by a short period of stabilisation after which the stage of preparation of the macrorupture commences. This ends with abrupt change of sign of the increment of volume deformation in all parts of structure. After that there is a macrodestruction.

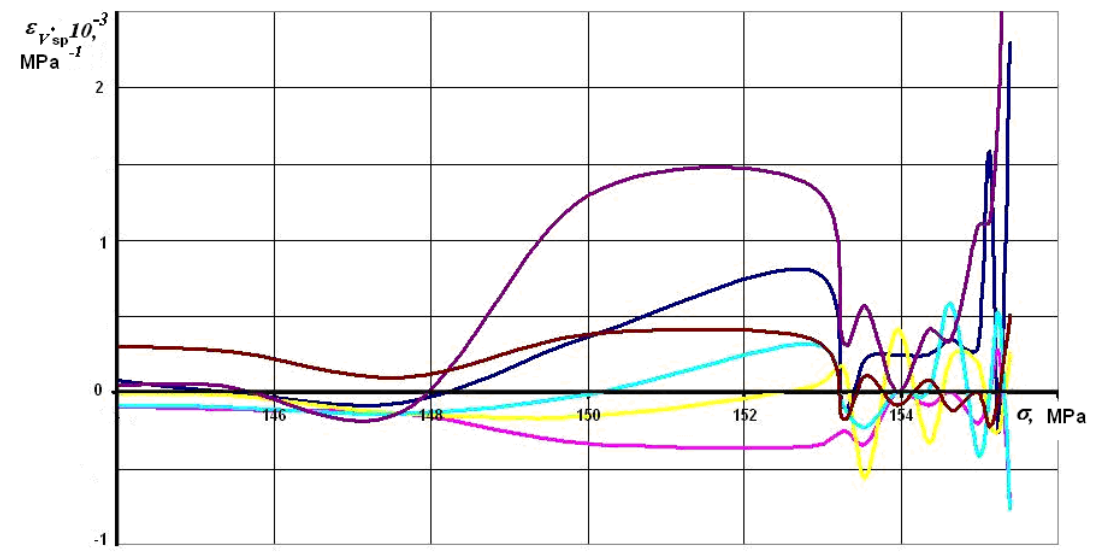

Figure 6 Relationship of rock sample deformation in prefailure stage of loading (granodiorite, sample No. 8)

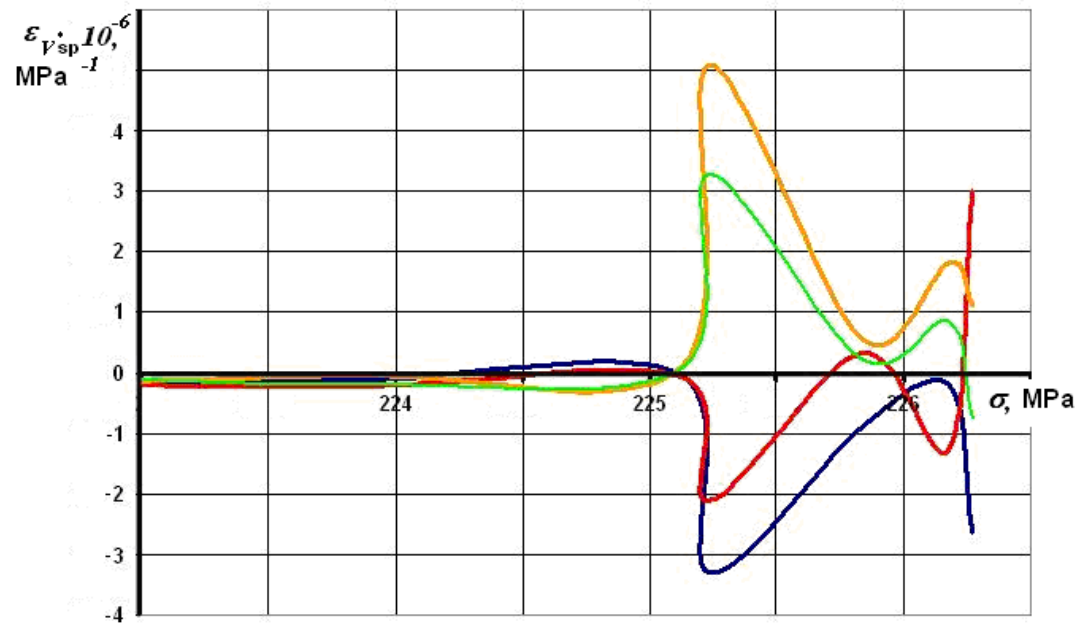

Figure 7 Relationship of rock sample deformation in prefailure stage of loading (granity-porphyry, sample No. 1, source part)

For three stages of deformation of the rock samples, their own precursors are identified:

- Material transform in a dilatancy condition (Stage I).

- Formation of zones of relative consolidation and dilatancy (Stage II).

- Change of the distribution character of the volume deformations increment (Stage III).

To these stages there are corresponding deformation precursors of rock failure (Figure 8):

- A long-term precursor - a point of intersection of the graph line of the volume specific deformation increment with an axis of pressure.

- An intermediate term precursor - the moment of simultaneous differing increments of volume specific deformation.

- A short-term precursor - the moment of sudden change of sign of the volume specific deformation increment. 


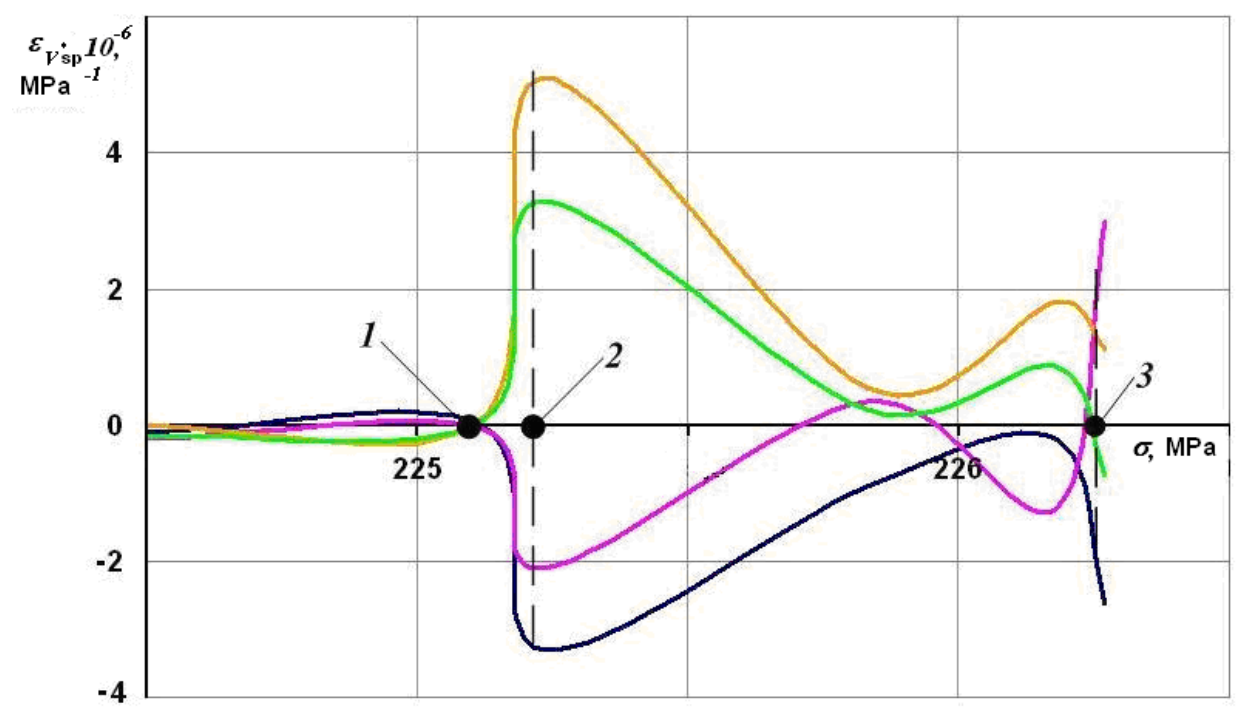

Figure 8 Deformation precursors of failure: 1 - long-term, 2 - immediate-term, 3 - short-term

To make the results reliable the experiment must have the following features:

- Measurements of linear deformations are needed for at least four local points located in the central part of the sample. The deviation of measuring position of devices from the central part of the sample should not exceed 0.1 part of its height.

- Fixing of loading and removal of the data with tensoresisters should be made with the minimum frequency of 2 measurements per second, and the speed of deformation changing in limits from 0.01 to $0.2 \mathrm{~mm} / \mathrm{sec}$.

- The loading device should be servocontrolled, providing constant stress rate.

For a more detailed study of the processes which are occurring in samples of rock in the prefailure loading area, independent research of the development of the source of destruction in the sample has been undertaken using the method of the acoustic emission thus allowing identification of the acoustic issue events connected with cracking in the sample.

\section{Experimental research of cracking regularities in rocks samples in the prefailure loading area}

The method of acoustic tomographical research of cracking laws in loading is well developed in experimental geomechanics (Lockner et al., 1991). In determining reliable deformation precursors, this method questions the reliability of the received results, and permits connection of the occurrence of the processes of meso- and macrocracks with deformation anomalies.

The primary goal of geoacoustic control is the fixing of the positions of the source of destruction in samples of rock in the prefailure loading area. For this purpose, gauges were mounted on the lateral surface of rock samples. The system used not less than four piezoelectric gauges. By the fixing the acoustic issue event by the system of gauges, this allows the calculation of the coordinates of each event according to the scheme of a relative arrangement of gauges set in a measuring complex, and the linear sizes of the sample.

By preparation for the experiment data acquisition equipment parameters, the following were preliminary set:

- Method of a signal fixation (on time of receipt/on achievement of peak amplitude).

- The maximum time of a signal increase.

- The maximum duration of a signal. 
- Speed of a sound distribution in a material.

- The linear sizes of the sample and a relative arrangement of gauges.

Realisation of a three-dimensional location is necessary to set the linear sizes on three axes. Thus, the model of the sample created in system, will have the parallel-piped form. It is necessary to consider the analysis of the received data. The obtained data allows not only defining coordinates of the source of destruction, but also its development in time that allows it to be compared with the results of the deformation data.

In the experiments, vibrating piezoelectric converters with fluctuations on a thickness were used, having a disk-shaped form, resonant frequency $1300 \mathrm{kHz}$ and diameter of $10 \mathrm{~mm}$ - MSAE-P500. These are highly sensitive gauges on using niobate of lithium with a broadband low noise preamplifier, working in a frequency range $50-1500 \mathrm{kHz}$ with cable connection (Figure 9). Characteristics of the gauges are presented in Table 1. Gauges were completely tight and protected from external electric interferences.

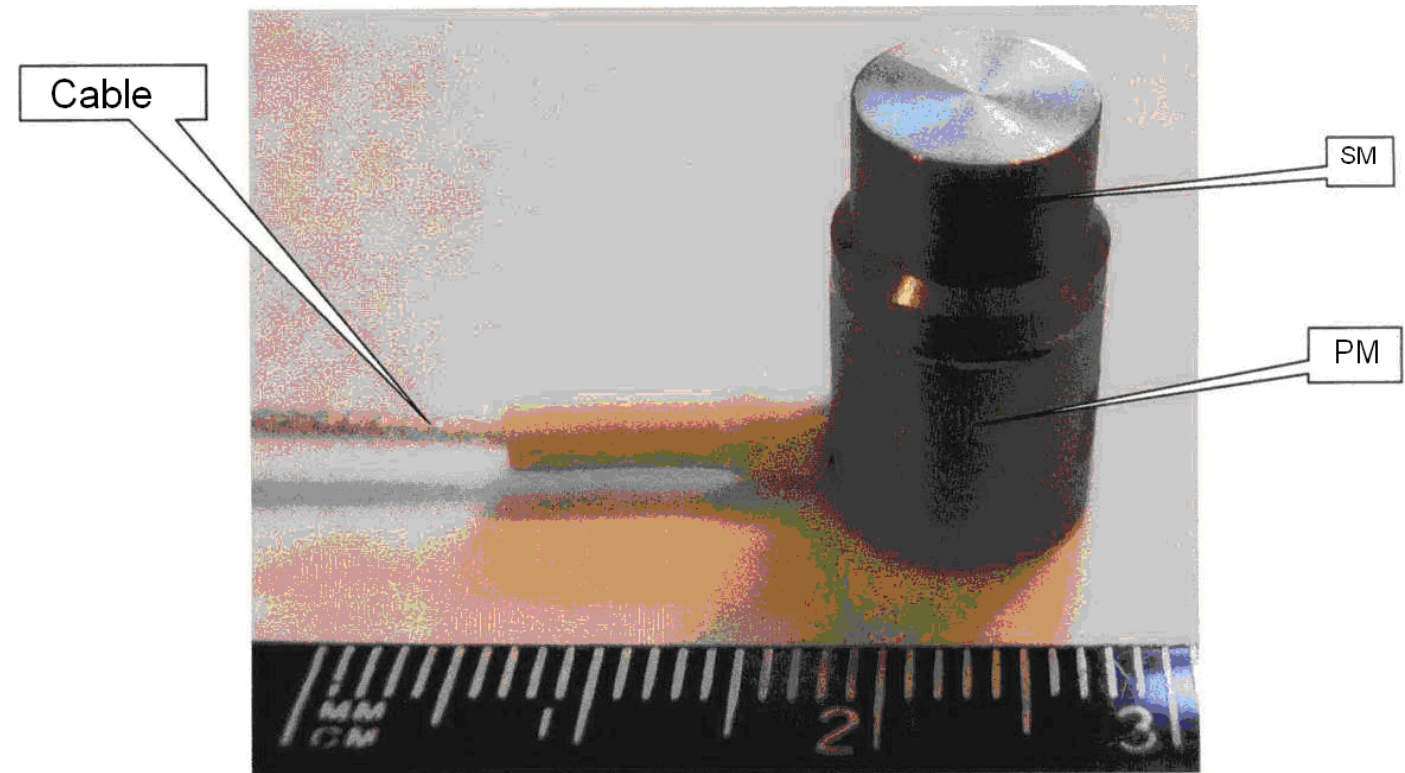

Figure 9 Gauge AE MSAE - P500: SM - sensitivity modules, PM - preamplification modules

Table 1 Main characteristics of gauges MSAE-P500

\begin{tabular}{lc}
\hline Characteristics & Meaning \\
\hline Peak sensitivity & $74 \mathrm{~dB}$ \\
Fringe of signal transmission & $50-1500 \mathrm{kHz}$ \\
Extremal resonance frequency & $1300 \mathrm{kHz}$ \\
Coefficient of preamplifier amplification & $27 \mathrm{~dB}$ \\
Mean square noise level & $<3 \mathrm{mV}$ \\
\hline
\end{tabular}

For data acquisition the measurement of the acoustic issue used a complex A-Line 32D. The complex carries out the non-destructive control of objects. The control purpose is the definition of the coordinates and tracking of the sources of acoustic issue in the cracks. The information received after processing is used for revealing and localising possible defects in objects (Figure 10). 


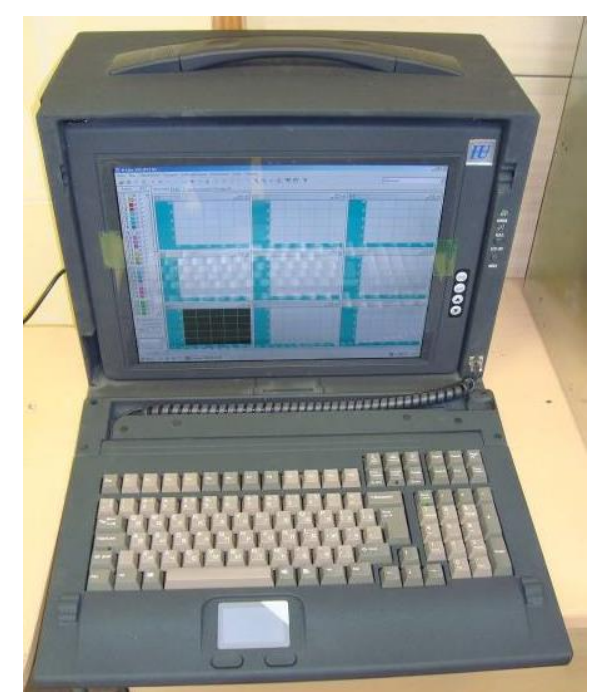

Figure 10 Acoustic emissive measurement complex A-Line 32D

Complex A-Line 32D represents a multichannel system of gathering and processing the acoustic emissive information received in real time from investigated object undertaken by the acoustic gauges.

To prepare for acoustic emissive measurements, it is necessary to provide, as much as possible, dense contact of piezoelectric gauges to a lateral surface of the sample. For this purpose, on a lateral surface of the sample of the cylindrical form, it is necessary to create an equal smooth surface, perpendicular to end faces.

For removal of emptiness between the gauge and the sample it is necessary to use greasing in a place of contact, and to provide uniform pressing of gauges to a lateral surface of the sample. Gauges are established in 6 points on a lateral surface of the sample symmetrically around the cylinder axis (Figure 11).

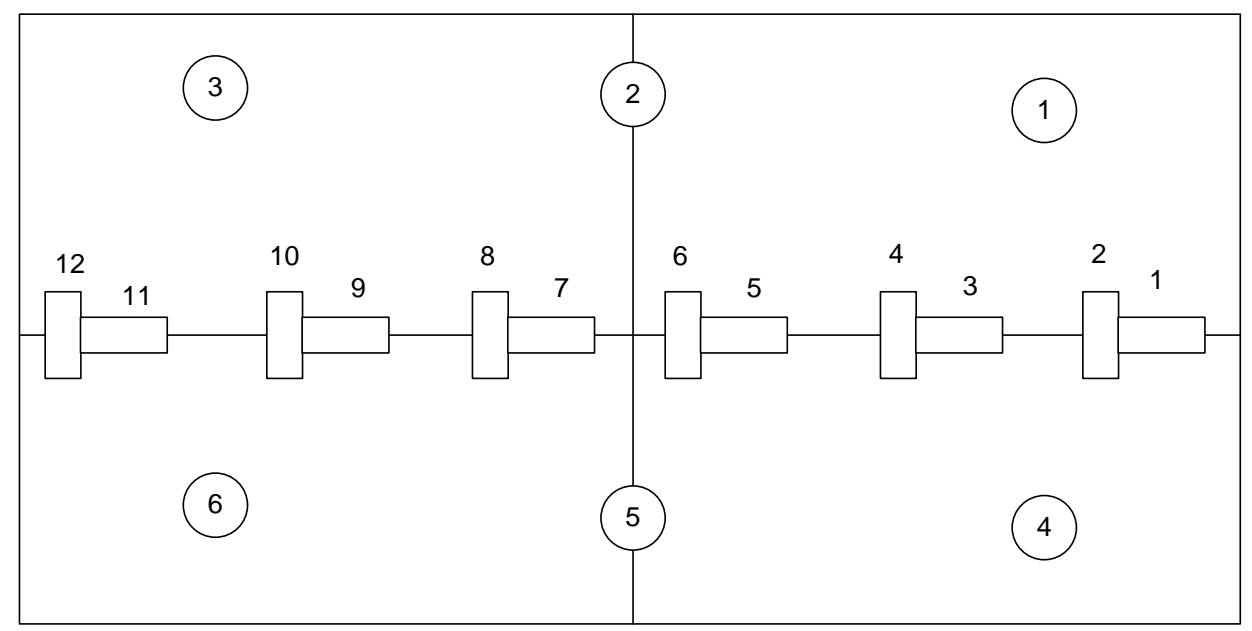

Figure 11 Scheme of acoustic emission gauges displacement

The determination of the elastic wave speeds in the axial and orthogonal directions was achieved using a generator of impulses on the piezoelectric converters. After the calculation of the speed of a wave, the speed between two opposite gauges was made taking into account the wave form. For an increase in the accuracy of the measurement speed, distribution of waves between various steams of gauges are calculated, then these values are compared.

All data from the acoustic gauges was fixed and processed using the acoustic emission measuring complex INTERUNIS A-Line 32D. The sample was placed in the test machine MTS-816 and loaded uniaxially, with the constant velocity up to failure (Figure 12). 


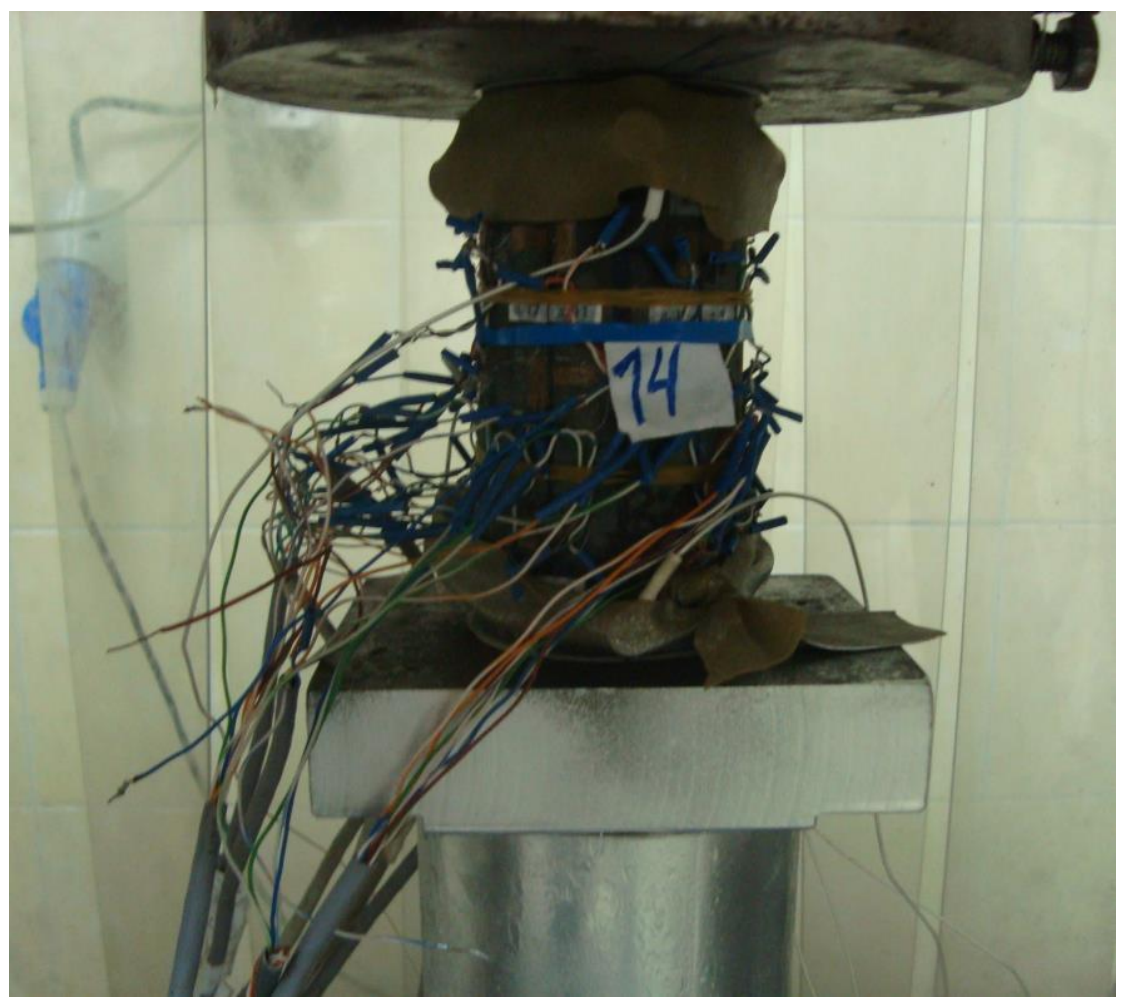

Figure 12 Rock sample in the loading process

The analysis of the received acoustic emission data allows tracking of the stages of the source development in the rock sample (Figure 13). At the first stage (Figure 13a), it is shown that, until time moment t1, defects are randomly distributed around the sample in regular intervals.

After time moment $\mathrm{t} 1$ and until time moment $\mathrm{t} 2$ there is a localisation of the destruction source. In the most part, signals concentrated in the area are located at the sub-centre of the sample (Figure 13b). Following time moment $\mathrm{t} 2$, there is a sharp increase in the quantity of registered events and macrorupture development begins (Figure 13c).

Thus, the authors note that the stages of deformation of the samples and the stages of formation of the destruction source practically coincide in time (Figure 14). It shows the dependence of deformation anomalies on the development of mesocracking structures within the sample.

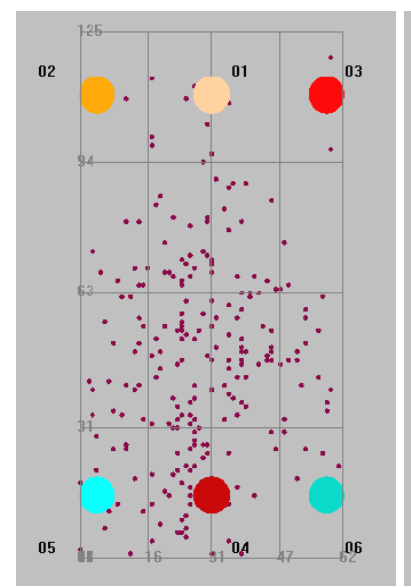

a)

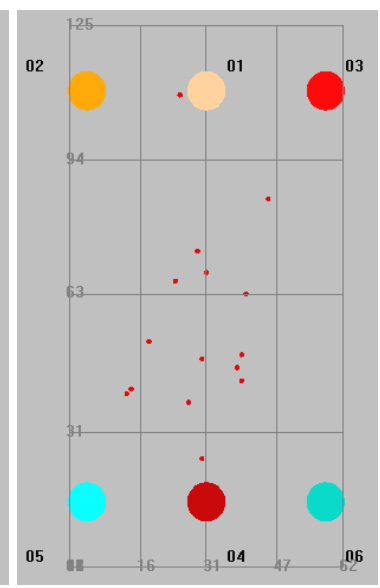

b)

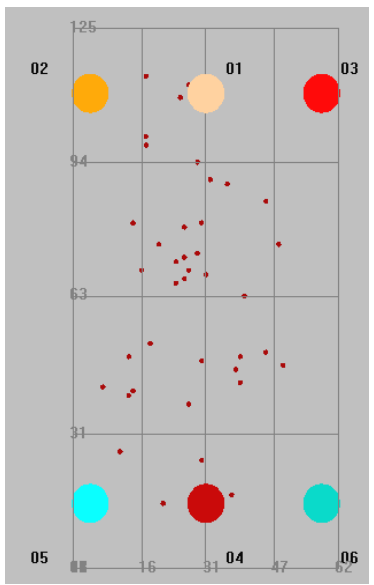

c)

Figure 13 Stages of destruction source development in rock sample: a) signals, registered in time period

0 -t1, chaotic stage of cracking; b) signals, registered in time period t1-t2, source 
localisation stage; c) signals, registered in time period t2-tк $\mathrm{p}$ stage of macrofailure source development

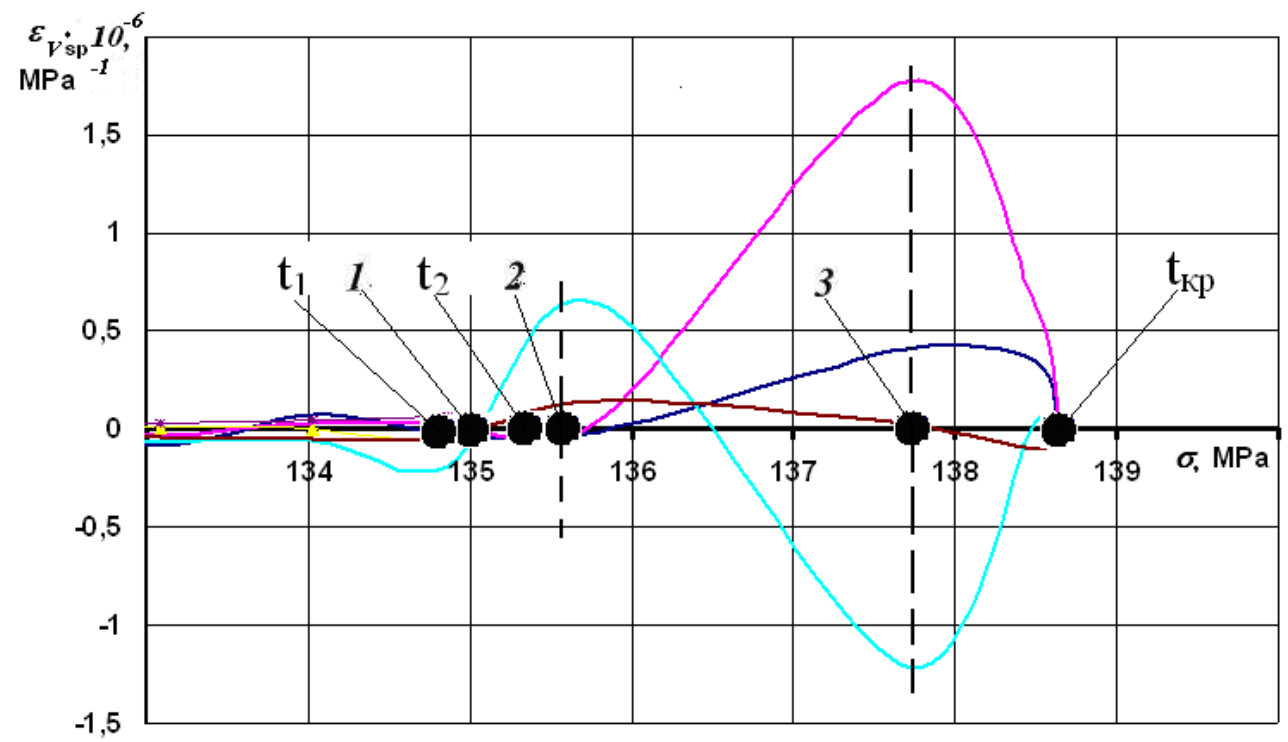

Figure 14 Precursors of rock samples failure. Deformational precursors: 1 - long-term, 2 - middleterm,

3 - short-term. Acoustic precursors: $t 1$ - moment of the coherent mesocracking process beginning,

t2 - moment of the mesocracking localisation process beginning, $t \kappa p$ - moment of the macrocracking process beginning

\section{Theoretical research of cracking regularities in rocks samples prefailure loading area}

The following mathematical model of self equilibrium stresses has been developed to describe the observed failure process (Guzev et al., 2005).

Deformation anomalies of a reversive type appear in the rock sample when loading $\sigma$ is above some critical value $\sigma>\sigma^{*}$. If $\sigma$ is less than $\sigma^{*}$, the stress-strain condition of the sample is described within the frames of the elasticity theory:

$$
\sigma_{i j}=\frac{E}{1+v}\left(\varepsilon_{i j}+\frac{v}{1-2 v} \varepsilon_{k k} \delta_{i j}\right),
$$

where:

$E \quad=\quad$ Young's modulus.

$v \quad=\quad$ Poisson's ratio.

When $\sigma$ is less then $\sigma^{*}$ the equations of balance for the rock sample in cylindrical coordinates look like:

$$
\begin{gathered}
\frac{\partial \sigma_{r r}}{\partial r}+\frac{1}{r} \frac{\partial \sigma_{r \varphi}}{\partial \varphi}+\frac{\partial \sigma_{r z}}{\partial z}+\frac{\sigma_{r r}-\sigma_{\varphi \varphi}}{r}=0 \\
\frac{\partial \sigma_{r \varphi}}{\partial r}+\frac{1}{r} \frac{\partial \sigma_{\varphi \varphi}}{\partial \varphi}+\frac{\partial \sigma_{\varphi z}}{\partial z}+\frac{2 \sigma_{r \varphi}}{r}=0
\end{gathered}
$$




$$
\frac{\partial \sigma_{r z}}{\partial r}+\frac{1}{r} \frac{\partial \sigma_{\varphi z}}{\partial \varphi}+\frac{\partial \sigma_{z z}}{\partial z}+\frac{\sigma_{r z}}{r}=0
$$

and boundary conditions of the task about the stress-strain condition of the cylindrical sample at uniaxial compression are formulated as:

$$
\begin{gathered}
\left.\sigma_{z z}\right|_{z= \pm h}=-\sigma,\left.\sigma_{z r}\right|_{z= \pm h}=0,\left.\sigma_{z \varphi}\right|_{z= \pm h}=0 \\
\left.\sigma_{r r}\right|_{r=R}=0,\left.\sigma_{r \varphi}\right|_{r=R}=0,\left.\sigma_{r z}\right|_{r=R}=0
\end{gathered}
$$

From the experimental results it follows (Figure 5) that the abnormal character of deformations in the loading area where $\sigma$ is more than $\sigma^{*}$ (deformations $x=0,5 \pi$ ) coincide with the order of sizes with subcritical deformations in area $\sigma$ less than $\sigma^{*}$. It allows us to connect the pressure $\Pi_{i j}$, corresponding to deformations, with the linear relationship similar on the algebraic structure to the Hooke's law for conditions of area where $\sigma$ it is less than $\sigma^{*}$ :

$$
\Pi_{i j}=\frac{E}{1+v}\left(E_{i j}+\frac{v}{1-2 v} E_{k k} \delta_{i j}\right)_{,(\mathrm{i}, \mathrm{j}=1,2,3)}
$$

where:

$E \quad=\quad$ Young's modulus.

$v \quad=\quad$ Poisson's ratio.

Formation of periodic mesocracking structures involves the occurrence of some new field of stress which generally depends on the type of cracking defects. As the sample is in balance, the forces defined by a field $T_{i j}$ should be compensated, this is called "self-counterbalanced". A compensating field is $\Pi_{i j}$, thus a full field of pressure in the sample $E_{i j}$ equals:

$$
\Sigma_{i j}=\Pi_{i j}+T_{i j}
$$

It is satisfied to equilibrium Equation (2) and boundary conditions (3). For fields $\Pi_{i j}$ and $T_{i j}$, it is also possible to note the corresponding equations of equilibrium:

$$
\frac{\partial \Pi_{i j}}{\partial x_{j}}=0 \quad \frac{\partial T_{i j}}{\partial x_{j}}=0
$$

and boundary conditions:

$$
\left.\Pi_{i j} n_{i}\right|_{\partial V}=-\left.T_{i j} n_{i}\right|_{\partial V}
$$

in that case:

$$
T_{i j}=2 \sigma_{0} l^{2} \varepsilon_{i p q} \varepsilon_{j m k} \frac{\partial \Gamma_{q m, p}}{\partial x_{k}}
$$

where:

$$
\begin{aligned}
& \varepsilon_{i p q}=\text { symbol of Levi-Chivity, constant. } \\
& \sigma_{0}, l=\text { have dimension of pressure and length accordingly. }
\end{aligned}
$$


Concrete kind of functions $\Gamma_{g m, p}$ depend on defective structure types, thus it is necessary to analyse the background of defect formation and dissipative processes in a material.

The problem statement for Equation (5) consists in the construction of the elastic field $\Pi_{i j}$. The deformations $E_{i j}$ corresponding to it coincide with the measured values the sample border in a discrete set of points.

The field of elastic pressure and deformations can be connected by linear relationships:

$$
\Pi_{i j}=A\left(E_{i j}+B E_{k k} \delta_{i j}\right)
$$

with the same coefficients $A, B$.

Without restriction of a generality of the parameters $A, B$, it is possible to select them as per the elasticity theory:

$$
A=\frac{E}{1+v}=2 \mu \quad B=\frac{v}{1-2 v}
$$

where:

$\mu \quad=\quad$ shear modulus.

As Equation (5) is linear, we will present a field $\Pi_{i j}$ in the form of the sum of the classical decision $\sigma_{i j}$ and sum field $\pi_{i j}$ :

$$
\Pi_{i j}=\sigma_{i j}+\pi_{i j}
$$

As the decision is under construction in the prefailure area, the loading level $\sigma=\sigma^{*}$ is the start point. Therefore, in Equation (3) for $\sigma_{i j}$, it is necessary to believe $\delta \sigma=\sigma-\sigma^{*}$ instead of $\sigma^{*}$. Additionally the first invariant $\pi_{k k}$ addressed is to be zero. Then the tensor $\pi_{i j}$ is connected with a corresponding tensor of deformation by a parity:

$$
\pi_{i j}=\mu\left(\frac{\partial a_{i}}{\partial x_{j}}+\frac{\partial a_{j}}{\partial x_{i}}\right)
$$

where:

$a_{i}=\quad$ components of a vector of the displacement, loadings counted from level $\sigma=\sigma^{*}$.

Components $a_{i}(i=1,2,3)$ are defined from the equations of balance which in cylindrical coordinates system look like:

$$
\Delta a_{r}-\frac{a_{r}}{r^{2}}-\frac{2}{r^{2}} \frac{\partial a_{\varphi}}{\partial \varphi}=0, \Delta a_{\varphi}-\frac{a_{\varphi}}{r^{2}}+\frac{2}{r^{2}} \frac{\partial a_{r}}{\partial \varphi}=0, \Delta a_{z}=0
$$

After the decision of system (12) in the form of a Fourier series on trigonometrical functions and carrying out of numerical calculations for experimental conditions at values of parameters of model: $v=0,26$, $E=1,7 * 10^{4} \mathrm{MPa}, x=0,5 \pi, h=5 \mathrm{~cm}, R=2,5 \mathrm{~cm}$, the authors received values of series coefficients:

$$
A_{21}^{(1)}=-3519 \cdot 10^{-6}, A_{41}^{(1)}=-29410 \cdot 10^{-6}, A_{11}^{(2)}=-1167 \cdot 10^{-6},
$$




$$
B_{21}^{(1)}=-700 \cdot 10^{-6}, B_{41}^{(1)}=885 \cdot 10^{-6}, B_{11}^{(2)}=1143 \cdot 10^{-6}
$$

Calculating present deformations, corresponding to Figure 5, and displaying them in comparison with the data of this experiment in Table 2, we can see that at full qualitative coincidence of the analytical and experimental results the maximum quantitative divergence of values of longitudinal deformations do not exceed $19 \%$.

Table 2 Results of comparison of the data theoretical and experimental research

\begin{tabular}{|c|c|c|c|c|c|c|c|c|}
\hline \multirow{3}{*}{ Parameter } & \multicolumn{8}{|c|}{$\begin{array}{l}\text { Longitudinal Deformations Number in Places of Gauges } \\
\text { (Makarov et al., 2008) }\end{array}$} \\
\hline & \multicolumn{2}{|c|}{$4-6$} & \multicolumn{2}{|c|}{$5-7$} & \multicolumn{2}{|c|}{$2-8$} & \multicolumn{2}{|c|}{$3-9$} \\
\hline & Exp. & Theory & Exp. & Theory & Exp. & Theory & Exp. & Theory \\
\hline $\begin{array}{l}\text { Longitudinal } \\
\text { deformations } \\
10-6\end{array}$ & $-1,067$ & -899 & 704 & 704 & -899 & -899 & 679 & 679 \\
\hline Deviation (\%) & \multicolumn{2}{|c|}{18.7} & \multicolumn{2}{|r|}{0.0} & \multicolumn{2}{|c|}{0.0} & \multicolumn{2}{|c|}{0.0} \\
\hline
\end{tabular}

\section{Conclusions}

The paper discuses experimental results illustrating anomalous deformation of rock samples under uniaxial compression, before the peak stress is reached. A system of trustworthy deformational precursors of the failure stage was developed. The system includes long-term, middle-term and short-term precursors. The observed criteria can be useful for prediction of the failure stage of mining pillars. Further development of this method for rock samples under triaxial compression will possibly allow formulating the system of failure precursors for conditions corresponding to the rock mass surrounding openings and for the earth crust.

\section{Acknow ledgement}

This paper has been written with support from FCP (Scientific staff of Russia for 2009-2013), the code number of the demand 2009-1.1-151-066-017.

\section{References}

Guzev, M.A. and Makarov. V.V. (2007) Deformation and failure of highly stressed rocks around the openings, Dalnauka Publishers, Vladivostok, $232 \mathrm{p}$.

Guzev, M.A., Makarov, V.V. and Ushakov, A.A. (2005) Modeling elastic behavior of compressed rock samples in the pre-failure zone, Journal of Mining Science, Springer New York Edit, Vol. 41, No. 6, pp. 497-509.

Lockner, D.A., Byerlee, J.D., Kuksenko, V., Ponomarev, A. and Sidorin, A. (1991) Quasi-static fault growth and shear fracture energy in granite, Nature - International Weekly Journal of Science, Vol. 350, No. 7, pp. 39-42.

Makarov, P.V. (2004) About the hierarchical nature of solids deformation and failure, Physical Mesomechanical, Vol. 7, No. 4, pp. 25-34.

Makarov, V.V., Ksendsenko, L.S., Opanasyuk, A.A. and Gnitienko, V.V. (2008) Periodical character of the high stressed rock samples, Mining Informational Analitycal Bulletin, No. 1, pp. 185-188.

Mechanics Test Systems (MTS) (09/2009) Rock and Concrete Mechanics Test Systems, Mechanics Test Systems (MTS), viewed 04 November 2011, http://www.mts.com/ucm/groups/public/documents/library/dev_002183.pdf.

Sagiya, T., Kanamori, H., Yagi, Y., Yamada, M. and Mori, J. (2011) Rebuilding Seismology, Nature - International Weekly Journal of Science, Vol. 473, pp. 146-148. 
